\title{
On charged impurity structures in liquid helium
}

\author{
A.A. Pelmenev ${ }^{1}$, I.N. Krushinskaya ${ }^{2}$, I.B. Bykhalo ${ }^{1}$, and R.E. Boltnev ${ }^{2}$ \\ ${ }^{I}$ Branch of Talroze Institute for Energy Problems of Chemical Physics, Russian Academy of Sciences, \\ Chernogolovka 142432, Russia \\ ${ }^{2}$ Joint Institute for High Temperatures, Russian Academy of Sciences, \\ Izhorskaya St. 13, Bd. 2, Moscow 125412, Russia \\ E-mail: boltnev@gmail.com
}

Received October 12, 2015, revised October 29, 2015, published online January 26, 2016

\begin{abstract}
The thermoluminescence spectra of impurity-helium condensates (IHC) submerged in superfluid helium have been observed for the first time. Thermoluminescence of impurity-helium condensates submerged in superfluid helium is explained by neutralization reactions occurring in impurity nanoclusters. Optical spectra of excited products of neutralization reactions between nitrogen cations and thermoactivated electrons were rather different from the spectra observed at higher temperatures, when the luminescence due to nitrogen atom recombination dominates. New results on current detection during the IHC destruction are presented. Two different mechanisms of nanocluster charging are proposed to describe the phenomena observed during preparation and warmup of IHC samples in bulk superfluid helium, and destruction of IHC samples out of liquid helium.

PACS: 78.60.Kn Thermoluminescence;

78.67.Bf Nanocrystals, nanoparticles, and nanoclusters;

36.40.Wa Charged clusters;

72.20.Jv Charge carriers: generation, recombination, lifetime, and trapping.
\end{abstract}

Keywords: nanoclusters, impurity-helium condensates, thermoluminescence, superfluid helium, stabilization of ions and radicals.

\section{Introduction}

Macroscopic impurity particles and systems formed directly in liquid helium-4 are known almost 70 years $[1,2]$. Such particles and systems have found many different applications: visualization of flows and quantum vortices in superfluid helium [2-4]; study of energy transfer phenomena in liquid helium [5]; development of new high-energy density materials [6-8]; investigation of tunneling reactions of hydrogen isotopes in impurity-helium condensates [9]; structural studies of rare gas, molecular deuterium and nitrogen nanoclusters [10-12]; study of cold neutron interaction with watergels $[13,14]$; synthesis of metallic nanowires in superfluid helium $[15,16]$. Laser ablation of metallic target in solid helium-4 allows to create so-called icebergs (helium crystals doped with metallic particles) remaining metastable below the melting curve $[17,18]$. It was shown that such icebergs contain rather high densities, $\sim 10^{15} \mathrm{~cm}^{-3}$, of positive ions and electrons [19].

In this paper we present new results on spectroscopic studies of thermoluminescence spectra of impurity-helium condensates (IHC) submerged in superfluid helium (He II) as well as the charge detection during the destruction of IHC samples. We explain the thermoluminescence of impurity-helium condensates submerged in superfluid helium as a result of neutralization reactions of thermoactivated electrons with nitrogen cations. To describe the phenomena observed during preparation and warm-up of IHC samples in bulk superfluid helium, and destruction of IHC samples out of liquid helium two different mechanisms of nanocluster charging are proposed.

\section{Experimental setup}

Impurity-helium condensates are highly porous materials formed, as shown in Fig. 1, by injection of a helium gas jet containing admixtures into superfluid helium at the $T=$ $=1.5 \mathrm{~K}$ [20]. Atoms of heavier noble gases as well as molecules of $\mathrm{N}_{2}, \mathrm{O}_{2}, \mathrm{H}_{2}$, and $\mathrm{NO}$ can be used as admixtures $(\sim 1 \%)$ to helium gas. A passage of a gas mixture through a radio frequency (rf) discharge area permits to obtain radicals, ions, metastable atoms and molecules by dissociation of impurity molecules and excitation of atoms. 

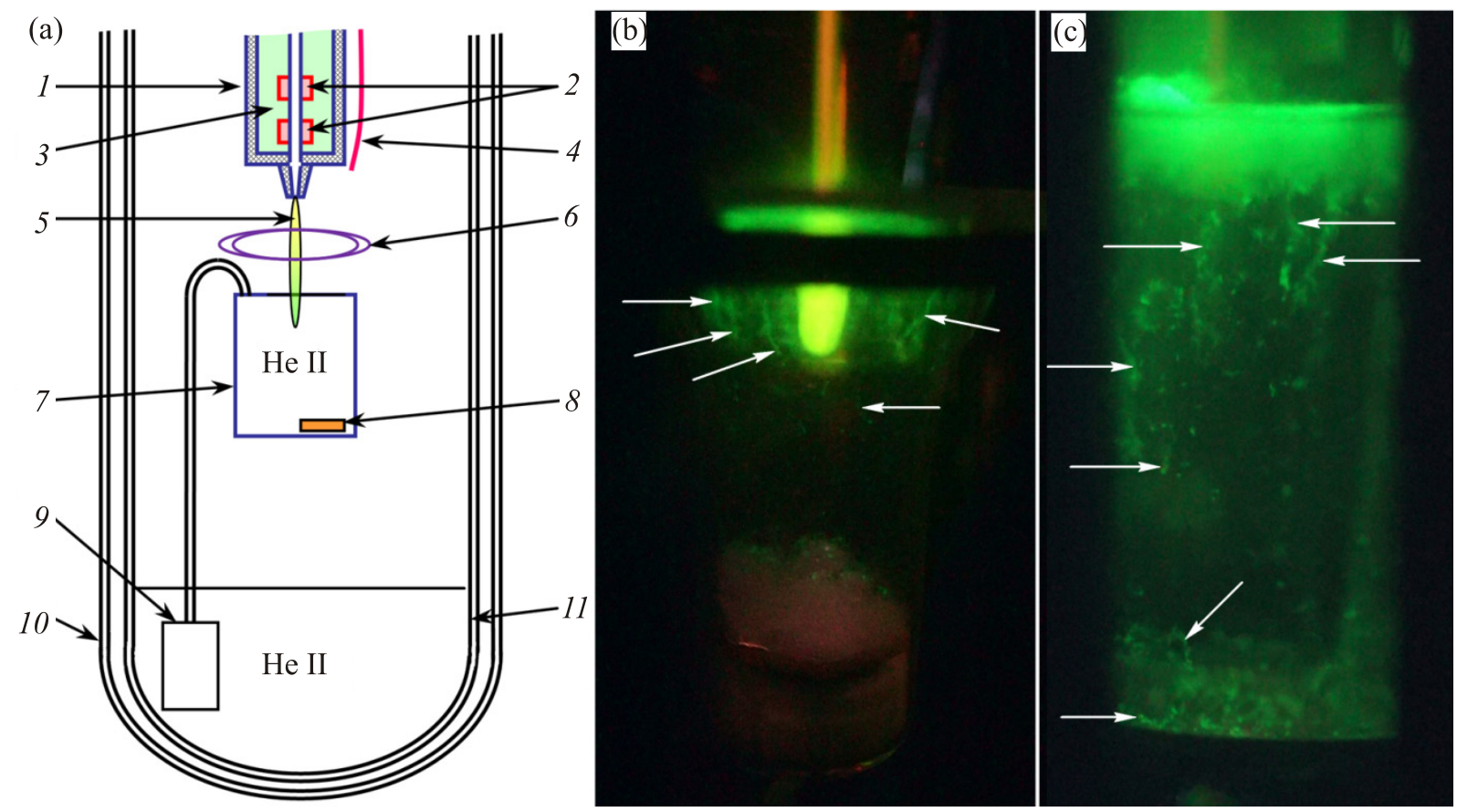

Fig. 1. (Color online) (a) Scheme of experimental setup for preparation and study of IHC samples: atom source (1); electrodes for rf discharge (2); liquid nitrogen (3); optical fiber (4); gas jet (5); ion collector (6); glass beaker filled with superfluid helium (7); thermometer (8); fountain pump (9); nitrogen glass dewar (10); helium glass dewar (11); (b) and (c) IHC sample preparation by condensation of the gas jet passed through an $\mathrm{rf}$ discharge (a gas mixture $\left[\mathrm{N}_{2}\right] /[\mathrm{He}]=1 / 100$ and $\left[\mathrm{N}_{2}\right] /[\mathrm{Xe}] /[\mathrm{He}]=1 / 1 / 400$, correspondingly). White arrows point to filament-like structures.

A scheme of the low-temperature part of experimental setup is shown in Fig. 1(a). While the jet transits the cold gas above the He II surface, impurity nanoclusters (with the characteristic size $\sim 3-6 \mathrm{~nm}$ ) form [12]. The nanoclusters penetrate through the superfluid surface and aggregate into an aerogel-like substance (Figs. 1(b) and 1(c)) with the impurity density of $\sim 10^{20}$ atom $/ \mathrm{cm}^{3}[10,12]$. A spectrometer AvaSpec-ULS2048XL-USB2 allowed us to detect emission within the spectral range from 200 to $1160 \mathrm{~nm}$ with the resolution $\approx 2.5 \mathrm{~nm}$. Emission from IHC sample under study was fed to the spectrometer by means of optical fibers and vacuum feedthrough. The relative spectral response of the detection system has been measured with the calibration light source AvaLight-DH-BAL-CAL. The pressure of helium vapor in the dewar within the range $0-10 \mathrm{kPa}$ was measured with a Rosemount gauge 3051TA2. A Lake Shore thermometer calibrated within the range 1.4-100 K was used for the temperature measurements. The ion currents accompanying the destruction of IHC samples were collected by a ring-shaped electrode (pos. 6 in Fig. 1(a)) connected directly to a picoammeter Keithley 6485 .

The green afterglow of the fresh samples (Fig. 1(b) and $1(\mathrm{c}))$ is due to so-called $\alpha$-group corresponding to the prohibited transition ${ }^{2} D-{ }^{4} S$ of nitrogen atom. The lifetime of $\mathrm{N}\left({ }^{2} D\right)$ atoms in matrices is greatly shortened, for example, from $\sim 4.4 \cdot 10^{4} \mathrm{~s}$ in the gas phase to $\sim 300 \mathrm{~s}$ and $\sim 40 \mathrm{~s}$ for neon and nitrogen matrices, correspondingly [21,22]. As it was shown earlier, luminescence of the IHC sample submerged in bulk He II can be stimulated by the temperature increase of $\sim 0.1 \mathrm{~K}$ of He II [23]. The fast decay stage (obeying to an exponential law with $\tau=15-40 \mathrm{~s}$ ) of the thermostimulated luminescence was followed by longlived decay obeying to a hyperbolic law with much longer $\tau$, up to $8 \cdot 10^{3} \mathrm{~s}$ [23]. In the present work we studied thermoluminescence spectra of IHC samples in He II during warm-up from 1.5 to $2.2 \mathrm{~K}$ (we will call such spectra as "cold" ones). A photon counting system was used to detect isothermal decay of very weak long-lived emission (the green afterglow) observed after sample preparation cessation and step-like temperature increase. The thermoluminescence spectra during destruction of IHC samples ("hot" spectra) were detected when the samples under study were out of liquid helium (it was evaporated from the beaker) at the temperature exceeding $2.2 \mathrm{~K}$.

\section{Experimental results}

\subsection{Macrostructure of IHC samples prepared with rf discharge applied}

First of all, we will describe the effect of rf discharge application on the macroscopic structure of the IHC samples. Small grains $(\sim 0.1 \mathrm{~mm})$ associate into bigger frag- 
ments on the beaker's edge and bottom when the gas jet was not undergone to the discharge. When the gas jet passes through a discharge area one can see also that long filaments (with the length $\sim 1 \mathrm{~cm}$ ) and flakes (with the diameter $\sim 1 \mathrm{~mm}$ ) form in the bulk He II (Figs. 1(b) and 1(c)). The flake and filament formation was not due to quantum vortices in superfluid helium because it was observed only when rf discharge was applied. Another effect of $\mathrm{rf}$ discharge application was observation how some fragments were dropping from the beaker edge. They rolled down along the beaker wall sticking to the wall like there was an electrostatic attraction between the IHC fragments and the beaker wall. The attraction was strong enough to deform the fragments. The IHC samples and their fragments are rather elastic and very compressible due to high porosity of the condensates [10].

\subsection{The green afterglow decay}

The temporal dependence of the $\alpha$-group intensity stimulated by a step-like temperature increase of the sample prepared from gas mixture $\left[\mathrm{N}_{2}\right] /[\mathrm{Ne}] /[\mathrm{He}]=$ $=1 / 100 / 5000$ is shown in Fig. 2 . The $\alpha$-group intensity decay is best fitted by hyperbolic law.

\subsection{Thermoluminescence spectra of IHC samples}

"Cold" and "hot" spectra observed during warm-up and destruction, respectively, of the IHC samples are shown in Fig. 3. The samples under study were prepared from gas mixtures $\left[\mathrm{N}_{2}\right] /[\mathrm{He}]=1 / 100$ and $\left[\mathrm{N}_{2}\right] /[\mathrm{Ne}] /[\mathrm{He}]=1 / 20 / 500$. The dominating features of the "cold" spectra were $\alpha$ group of $\mathrm{N}$ atom and the Vegard-Kaplan bands of $\mathrm{N}_{2}$ molecule $\left(A^{3} \Sigma_{u}^{+}, \quad v^{\prime}=0 \rightarrow X^{1} \Sigma_{g}^{+}, \quad 2 \leq v^{\prime \prime} \leq 12\right.$ transitions). New additional features were observed in the "hot" spectra: the $\beta$-group of $\mathrm{O}$ atom $\left({ }^{1} S_{-}{ }^{1} D\right.$ transition), the $\delta$-line of $\mathrm{N}$ atom $\left({ }^{2} P_{-}{ }^{2} D\right.$ transition), as well as $\beta$ - and M-bands of NO molecule $\left(B^{2} \Pi-X^{2} \Pi\right.$ and $a^{4} \Pi-X^{2} \Pi$ transitions), correspondingly, in nitrogen-helium and nitrogen-neon-helium samples. The "hot" spectra shown in Fig. 3 were detected at $T=13-15 \mathrm{~K}$.

\subsection{Currents accompanying destruction of IHC samples}

The currents detected during destruction of IHC samples containing stabilized radicals have revealed existence of charges in the condensates [24,25]. To find out a sign of charge carriers we have modified the geometry of electrodes (Fig. 4(a)). The stainless steel collector has been set above the beaker with the sample under study (Figs. 1(a) and 4(a)) and directly connected to the "high" input of a picoammeter. The second, "low", input was grounded. Such a scheme excludes the same sign of signals due to negative and positive charges detected, respectively, by anode and cathode. Typical current signals detected during destruction of nitrogen-helium sample are shown in Fig. 4(c). The unipolar current peaks reflect the signs and values of the charges accumulated by sample fragments touching the ion collector (anode). Bipolar signals correspond to currents induced on the collector by passing charged particles. A photo in Fig. 4(b) shows the destruction process of the nitrogen-neon-helium samples. Scatters of burning sample fragments from the beaker accompany the explosions during the sample destruction. Due to rather long exposure time $\sim 0.1 \mathrm{~s}$, one can see trajectories of some sample fragments leaving the beaker during the sample explosions. In addition to the burning sample and fragments flying away, one can see in the beaker a bright green flash — a cloud of glowing dust.
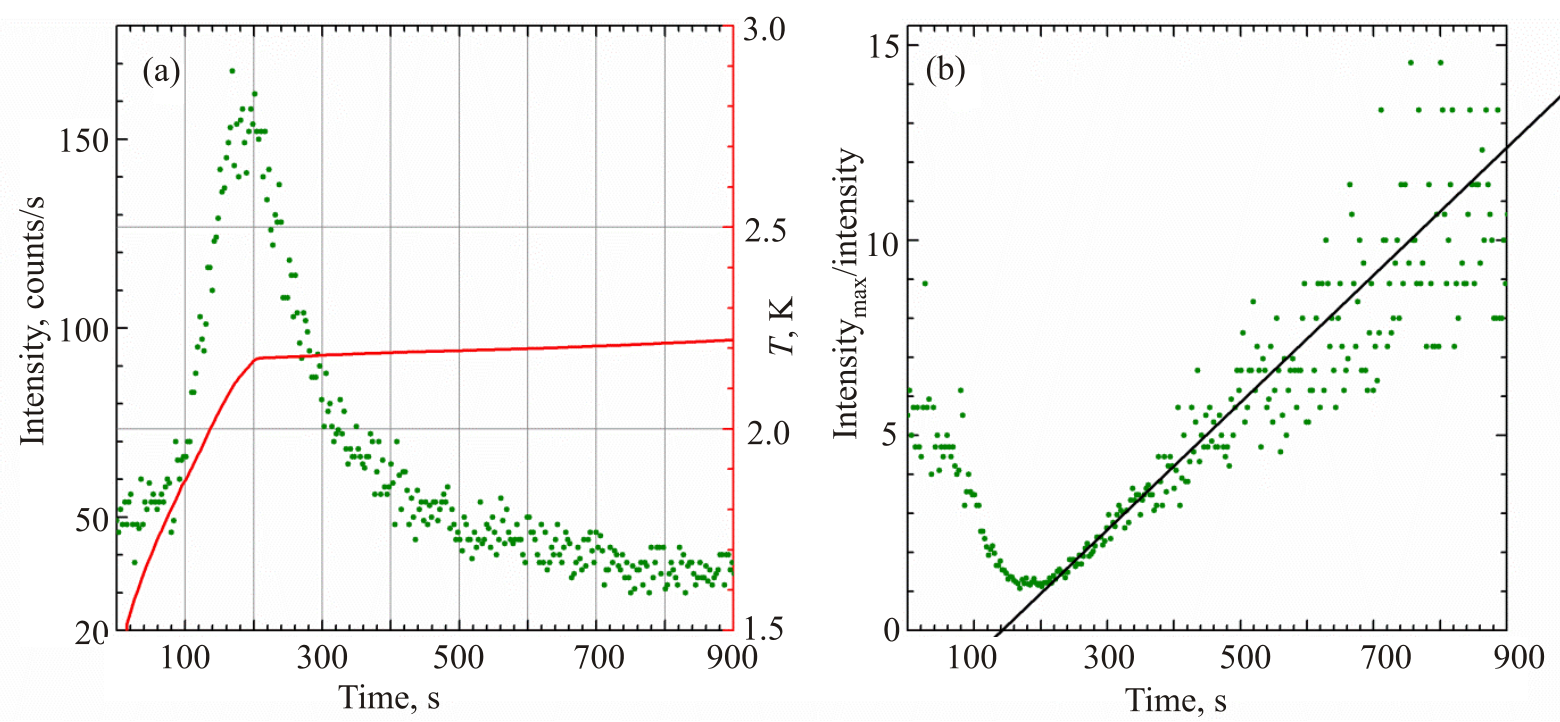

Fig. 2. (Color online) (a) Temporal dependences of the temperature (red line) and the thermostimulated $\alpha$-group emission (green dots) of the sample prepared from a gas mixture $\left[\mathrm{N}_{2}\right] /[\mathrm{Ne}] /[\mathrm{He}]=1 / 100 / 5000 ;$ (b) the fit of the emission decay as $I^{-1} \sim t($ line). 

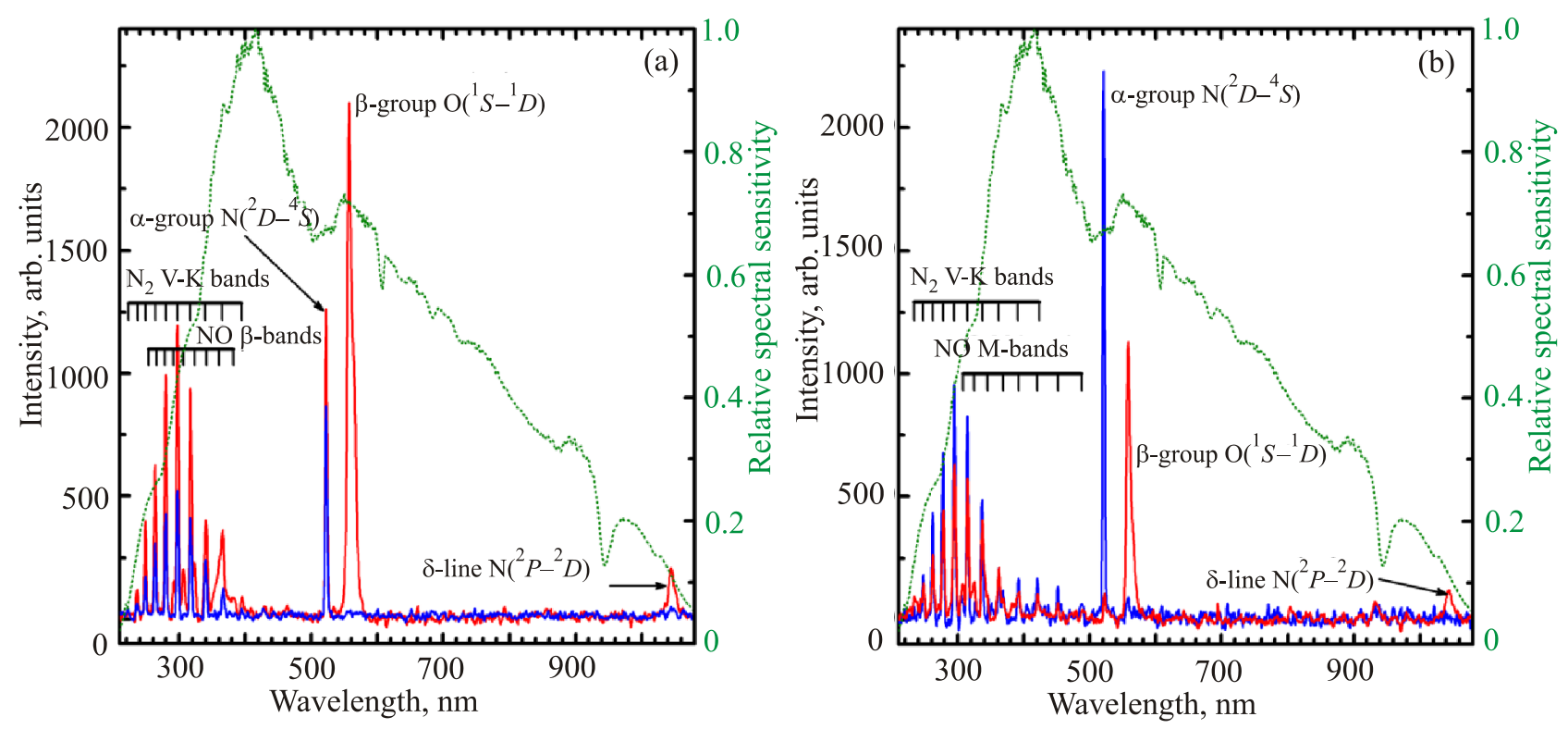

Fig. 3. (Color online) A comparison between the thermoluminescence spectra during warm-up and destruction (blue and red lines, respectively) of the IHC samples prepared from: (a) gas mixture $\left[\mathrm{N}_{2}\right] /[\mathrm{He}]=1 / 100$; (b) gas mixture $\left[\mathrm{N}_{2}\right] /[\mathrm{Ne}] /[\mathrm{He}]=1 / 20 / 500$. The spectral response of the detection system is shown by green dotted line.

\section{Discussion}

\subsection{Formation of charged nanoclusters and macroscopic structure of impurity-helium condensates}

The intense formation of filaments during preparation of impurity-helium condensates due to application of radiofrequency discharge (Fig. 1(b) and (c)) agrees very well with the idea of ion presence in impurity nanoclusters. The appearance of charged nanoclusters makes their interaction stronger and anisotropic. The filament formation is not the result of the coalescence of impurity grains and flakes due to quantum vortices $[15,26]$ because such filaments have never been observed during preparation of impurity-helium condensates without of radiofrequency discharge. We suggest that electrons and ions are trapped in impurity nanoclusters during cluster growth in a cold gas jet. We observed spectra of ions $\mathrm{N}_{2}^{+}$in the jet just above the He II surface during IHC sample preparation [27]. A possibility of creation of nitrogen cations $\mathrm{N}^{+}, \mathrm{N}_{2}^{+}, \mathrm{N}_{3}^{+}$, and $\mathrm{N}_{4}^{+}$, in helium-nitrogen plasma is rather high mainly due to the efficient Penning ionization by helium metastable species $\left(\mathrm{He}^{+}, \mathrm{He}^{*}\right.$, and $\mathrm{He}_{2}^{*}$ ) [28-31], particularly in cold (at $T<$ $<100 \mathrm{~K})$ afterglow, where ions $\mathrm{N}_{n}^{+}$with $1 \leq n \leq 9$ had been observed [32]. We should also keep in mind that the nitrogen atoms, molecules, and nanoclusters moving along
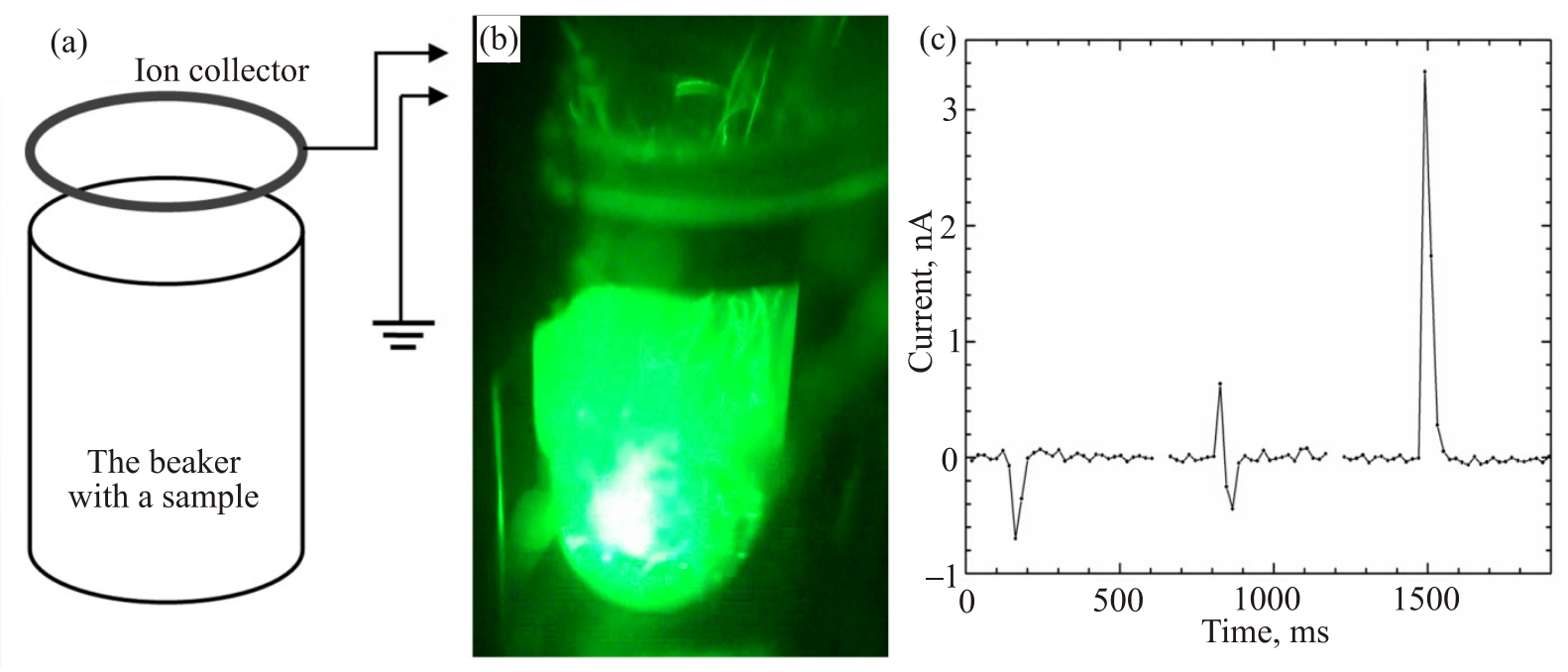

Fig. 4. (Color online) Detection of current pulses during destruction of IHC samples: (a) disposition of the ion collector and the beaker containing an IHC sample; (b) explosive destruction of an IHC sample; (c) the current pulses detected during destruction of the sample prepared from $\left[\mathrm{N}_{2}\right] /[\mathrm{He}]=1 / 100$ gas mixture. 
the jet are still irradiated with VUV photons from helium plasma within the discharge area (Fig. 1). Such photons are also able to ionize nitrogen atoms and molecules [33]. Therefore, the presence of charged nanoclusters in IHC samples prepared with rf discharge application should be quite obviously.

We have estimated the charge values of sample fragments from the current amplitudes (Fig. 4(c)): the current of $\sim \mathrm{nA}$ produces the voltage $\sim 10 \mu \mathrm{V}$ (the input resistance of the picoammeter is equal to $10 \mathrm{kOhm}$ for the work range below $20 \mathrm{nA}$ ). To charge the measurement circuit capacitance $\sim 100 \mathrm{pF}$ up to the potential of $10 \mu \mathrm{V}$ we need $\sim 1 \mathrm{fC}$ or about of $10^{4}$ elementary charges. Detection of IHC fragments carrying $\sim 10^{4}$ elementary charges was very surprising for us. Definitely, the growth of impurity clusters around ionic centers and capturing electrons by the clusters in the cold jet can't be a reason of formation of macroscopic aggregations consisting of nanoclusters bearing unipolar electric charges. We suggest a capture of electrons from a glass beaker surface by excited metastable $\mathrm{N}_{2}\left(A^{3} \Sigma\right)$ molecules might be responsible for the charging of IHC samples. Formation of $\mathrm{N}_{2}^{-}\left(X^{2} \Pi_{g}\right)$ through a capture of electrons from dielectric and metallic surfaces by metastable $\mathrm{N}_{2}\left(A^{3} \Sigma\right)$ molecules has been studied rather well [34-36]. The destruction of IHC sample starts with a sublimation of the helium shells surrounding impurity nanoclusters and preventing the recombination of nitrogen atoms stabilized on the nanocluster surfaces. Nitrogen atom recombination produces mainly metastable molecules $\mathrm{N}_{2}\left(A^{3} \Sigma\right)$ observable due to intense luminescence $[24,25,27]$. Then $\mathrm{N}_{2}\left(A^{3} \Sigma\right)$ molecules contacting with the beaker wall capture the electrons. That is why the sample surface next to the beaker is charging negatively. When the sample loses its integrity because of explosions, the charged fragments fly away due to Coulomb repulsion (Fig. 4(b)) and some of them are detected by the ion collector (Fig. 4(c)).

Therefore, we can explain the IHC structure modification and the results on current detections during IHC sample destructions by two different mechanisms of nanocluster charging. The first one consists in trapping positive ions and electrons by nanoclusters growing in the cold jet. It forms quasineutral IHC samples. The second mechanism provides a charge separation: nanoclusters capture electrons from the contacting surfaces due to the peculiarity of $\mathrm{N}_{2}\left(A^{3} \Sigma\right)$ molecule interaction with dielectric and metallic surfaces.

During IHC sample destruction we can see, beside of the burning sample, some fragments flying away and a cloud of glowing ultrafine particles. Keeping in mind that some big and ultrafine particles are charged we can define the whole system as cryogenic dusty plasma. From this point of view, a behavior of such systems in external electric field is of great interest.

\section{2. "Cold" and "hot" thermoluminescence spectra from IHCS}

The initial point of this work was the principal distinction between the "cold" and "hot" spectra observed from nitrogen-helium and nitrogen-neon-helium condensates. This distinction along with last results on currents detected during destruction of IHCs $[24,25]$ had allowed us to suggest existence of two different mechanisms responsible for "cold" and "hot" luminescence from IHCs.

The earlier explanation of the thermostimulated luminescence of IHC samples submerged in He II was related to the model of van der Waals clusters consisting of single impurity atom or molecule surrounded by a shell of localized helium atoms [23]. The key point was the extremely weak perturbation of the helium shell on the central metastable $\mathrm{N}\left({ }^{2} D\right)$ atom. The lifetime of such $\left.\mathrm{N}^{2} D\right)$ atoms was expected to be close to its gas phase value $\sim 10^{4} \mathrm{~s}$. The green emission on ${ }^{2} D_{-}{ }^{4} S$ transition was stimulated by "heavy" particle $\left(\mathrm{N}_{2}\right.$ molecule, atoms of neon, argon, or $\mathrm{Kr})$ approaching $\mathrm{N}\left({ }^{2} D\right)$ atom. The high sensitivity of the IHC samples to the temperature changes has been explained by the low value (about of $40 \mathrm{~K}$ ) of the energy needed for helium shell rearrangement and meeting of $\mathrm{N}\left({ }^{2} D\right)$ atom and "heavy" particle [23]. Nevertheless, the model did not allow us to explain the photostimulated luminescence of IHC samples submerged in bulk He II. Moreover, the model was not confirmed by structural studies of IHCs revealed nanoclusters as building units of the condensates [10-12].

It is well known that each temperature increase of cryocrystals of rare gases and molecular nitrogen is followed by thermal mobilization of the electrons trapped in shallow traps $[24,37,38]$. We suggest that the electrons are localized in traps of varying depth (with the different activation energy). Some impurity centers as well as different structural defects of cryocrystals and clusters can be such electron traps. There are very broad distributions of electron traps in cryocrystals and clusters on the activation energy: the activation energies of 3.5 and $20 \mathrm{meV}$ were determined for structure defects in neon nanoclusters [23] and cryocrystals [39], respectively, while the electron affinity energy of $\mathrm{O}\left({ }^{3} P\right)$ atom is of $1.46 \mathrm{eV}$ [40]. Therefore, thermo- or photostimulated electrons leave the traps and initiate neutralization reactions. It was shown that such electrons are responsible for luminescence observed in cryocrystals $[39,41]$. We suggest similar processes were observed in cryocondensates [42-44] and impurity nanoclusters [23] at temperatures below $10 \mathrm{~K}$ when the atoms are still immobile in solids.

Our analysis of the literature shows that the products of neutralization reactions of $\mathrm{N}^{+}$and $\mathrm{N}_{2}^{+}$with electrons, and dissociative recombination reactions of $\mathrm{N}_{3}^{+}$, and $\mathrm{N}_{4}^{+}$with electrons are mainly $\left.\mathrm{N}^{2} D\right)$ atoms and $\mathrm{N}_{2}$ molecules in the metastable lowest singlet $\left(a^{\prime} \Sigma_{u}^{-}\right)$and triplet $\left(A^{3} \Sigma_{u}^{+}\right)$ 
states [45-50]. The bands corresponding to the transition $a^{\prime 1} \Sigma_{u}^{-} \rightarrow X^{1} \Sigma_{g}^{+}$were out of the spectrometer work range.

The absence of the $\beta$-group emission in the "cold" spectra reflects the fact that the numbers of ions $\mathrm{O}^{+}$stabilized in neon and nitrogen nanoclusters are much less comparing to nitrogen cations. Now, we should mention some additional pathways of formation of excited $\mathrm{N}$ and $\mathrm{O}$ atoms in solid nitrogen matrix [22]:

$$
\begin{aligned}
& \mathrm{N}_{2}\left(A^{3} \Sigma_{u}^{+}\right)+\mathrm{N}\left({ }^{4} S\right) \rightarrow \mathrm{N}\left({ }^{2} D\right)+\mathrm{N}_{2}\left(X^{1} \Sigma_{g}^{+}\right), \\
& \mathrm{N}_{2}\left(A^{3} \Sigma_{u}^{+}\right)+\mathrm{N}\left({ }^{4} S\right) \rightarrow \mathrm{N}\left({ }^{2} P\right)+\mathrm{N}_{2}\left(X^{1} \Sigma_{g}^{+}\right), \\
& \mathrm{N}_{2}\left(A^{3} \Sigma_{u}^{+}\right)+\mathrm{O}\left({ }^{3} P\right) \rightarrow \mathrm{O}\left({ }^{1} S\right)+\mathrm{N}_{2}\left(X^{1} \Sigma_{g}^{+}\right) .
\end{aligned}
$$

There were no the features of $\mathrm{N}\left({ }^{2} P\right)$ and $\mathrm{O}\left({ }^{1} S\right)$ atoms (the $\delta$-line and the $\beta$-group, correspondingly) detected in the "cold" spectra (Fig. 3). We suggest this fact is due to inefficiency of the energy migration $\mathrm{N}_{2}\left(A^{3} \Sigma_{u}^{+}\right)+$ $+\mathrm{N}_{2}\left(X^{1} \Sigma_{g}^{+}\right) \rightarrow \mathrm{N}_{2}\left(X^{1} \Sigma_{g}^{+}\right)+\mathrm{N}_{2}\left(A^{3} \Sigma_{u}^{+}\right)$within nitrogen nanoclusters because of high density of structure defects [10]. This assumption is supported by identical "cold" spectra detected from nitrogen-neon-helium sample. There is no energy transfer possible from excited nitrogen molecule to neither $\mathrm{N}$ nor $\mathrm{O}$ atom isolated in a neon matrix. So, "cold" spectra contain the features only of neutralization reaction products.

As one can see, the features of "cold" spectra can be well explained by recombination of nitrogen cations and electrons trapped in nitrogen and neon nanoclusters. The high sensitivity of IHCs to temperature changes is a consequence of the shallow traps (with the depth of $\sim \mathrm{meV}$ ) formed by structural defects. The suggested mechanism of thermoluminescence allows us also to explain an origin of photostimulated emission of IHC samples submerged in He II [23]. Electrons can be releazed from the traps by photons of appropriate energy and promoted into the conduction band [51]. Such photomobilized electrons participate in exoelectron emission [51-53], react with holes producing luminescence $[42,54,55]$ and anomalous low-temperature post-desorption from the cryocrystal surfaces $[45,56]$.

The thermoluminescence mechanism proposed agrees well with the experimental results of the ESR studies of IHCs containing nitrogen atoms: the number of stabilized $\mathrm{N}\left({ }^{4} S\right)$ atoms during the warm-up of the samples in bulk He II remains almost constant [8].

Therefore, we suggest that neutralization reactions are responsible for luminescence of IHC samples submerged in He II ("cold" spectra), while recombination of $\left.\mathrm{N}^{4} S\right)$ atoms dominates as energy source for thermoluminescence ("hot" spectra) of "dry" IHC samples at higher temperatures.

The hyperbolic decay of the long-lived afterglow (Fig. 2(b)) corresponds to electron release from the traps uniformly distributed on the depths [57]. Thus, the thermoluminescence observed in IHC samples submerged in He II can be explained by electrons releazed from the traps with very broad distribution of the activation energy $(\sim \mathrm{meV}$ in the structure defects and $\sim \mathrm{eV}$ at atoms with large positive electron affinity energy) in impurity nanoclusters. The temperature decrease causes fast $\alpha$-group decay with the characteristic times much shorter than the lifetimes of metastable $\mathrm{N}\left({ }^{2} D\right)$ atoms stabilized in corresponding matrices [23].

\section{Conclusions}

In this paper, we have presented new experimental results of optical spectroscopy and current detection studies of impurity-helium condensates. The phenomena observed during formation (growth of impurity filaments and sticking of IHC fragments to a glass surface) and destruction (scattering of fragments and current pulse detection) of impurity-helium condensates, as well as the distinctions between the "cold" and "hot" spectra had allowed us to propose existence of two different mechanisms of charging impurity nanoclusters.

1. Thermoluminescence spectra of nitrogen-helium and nitrogen-neon-helium condensates submerged in superfluid helium had been observed for the first time.

2. The phenomena observed during impurity-helium condensate formation can be explained by presence of ions trapped in impurity nanoclusters. Electrons and positive ions are trapped by the nanoclusters growing in cold helium gas jet passed through a radiofrequency discharge.

3. The luminescence of IHC samples submerged in bulk He II is caused by neutralization reactions with participation of thermo- and photoactivated electrons and nitrogen cations stabilized in impurity nanoclusters.

4. Another mechanism of the charged nanoclusters formation is proposed to describe the phenomena observed during the destruction of IHC samples: intense recombination of nitrogen atoms during the IHC sample destruction produces excited molecules $N_{2}\left(A^{3} \Sigma\right)$ which capture electrons from a substrate and charge the sample fragments up to $10^{4}$ elementary charges.

5. Charged big fragments and ultrafine particles form systems which can be defined as cryogenic dusty plasma. Such systems in gas and liquid phases of helium-4 are of great interest for studying at temperatures of $1-10 \mathrm{~K}$.

\section{Acknowledgments}

The work has been done under support of the Russian Science Foundation (grant \#14-50-00124) in Joint Institute for High Temperatures.

1. P. Savich and A. Shalnikov, J. Phys. USSR 10, 229 (1946).

2. K.L. Chopra and J.B. Brown, Phys. Rev. 108, 157 (1957).

3. D.Y. Chung and P.R. Critchlow, Phys. Rev. Lett. 14, 892 (1965).

4. T.A. Kitchens, W.A. Steyert, and R.D. Taylor, Phys. Rev. Lett. 14, 942 (1965). 
5. J. Jortner, L. Meyer, S.A. Rice, and E.G. Wilson, Phys. Rev. Lett. 12, 415 (1964).

6. E.B. Gordon, L.P. Mezhov-Deglin, O.F. Pugachev, and V.V. Khmelenko, JETP 46, 502 (1977) [ZhETF 73, 952 (1977)].

7. E.P. Bernard, R.E. Boltnev, V.V. Khmelenko, and D.M. Lee, J. Low Temp. Phys. 134, 199 (2004).

8. R.E. Boltnev, I.N. Krushinskaya, A.A. Pelmenev, E.A. Popov, D.Yu. Stolyarov, and V.V. Khmelenko, Fiz. Nizk. Temp. 31, 723 (2005) [Low Temp. Phys. 31, 547 (2005)].

9. E.B. Gordon, A.A. Pelmenev, O.F. Pugachev, and V.V. Khmelenko, JETP Lett. 37, 282 (1983) [Pis'ma ZhETF 37, 237 (1983)].

10. V. Kiryukhin, B. Keimer, R.E. Boltnev, V.V. Khmelenko, and E.B. Gordon, Phys. Rev. Lett. 79, 1774 (1997).

11. E.P. Bernard, R.E. Boltnev, V.V. Khmelenko, V. Kiryukhin, S.I. Kiselev, and D.M. Lee, Phys. Rev. B 69, 104201 (2004).

12. N.V. Krainyukova, R.E. Boltnev, E.P. Bernard, V.V. Khmelenko, D.M. Lee, and V. Kiryukhin, Phys. Rev. Lett. 109, 245505 (2012).

13. L.P. Mezhov-Deglin, V.B. Efimov, A.V. Lokhov, E.V. Lychagin, A.Y. Muzychko, V.V. Nesvizhevskii, and A.V. Strelkov, J. Low Temp. Phys. 148, 883 (2007).

14. V.B. Efimov, L.P. Mezhov-Deglin, C.D. Dewhurst, A.V. Lokhov, and V.V. Nesvizhevsky, Adv. High Energy Physics 1, 808212 (2015).

15. P. Moroshkin, V. Lebedev, B. Grobety, C. Neururer, E.B. Gordon, and A. Weis, EPL 90, 34002 (2010).

16. E.B. Gordon, A.V. Karabulin, V.I. Matyushenko, V.D. Sizov, and I.I. Khodos, Fiz. Nizk. Temp. 36, 740 (2010) [Low Temp. Phys. 36, 590 (2010)].

17. P. Moroshkin, A. Hofer, S. Ulzega, and A. Weis, Nat. Phys. 3, 786 (2007).

18. V. Lebedev, P. Moroshkin, and A. Weis, Fiz. Nizk. Temp. 35, 391 (2009) [Low Temp. Phys. 35, 299 (2009)].

19. P. Moroshkin, V. Lebedev, and A. Weis, Phys. Rev. Lett. 102, 115301 (2009).

20. E.B. Gordon, L.P. Mezhov-Deglin, and O.F. Pugachev, JETP Lett. 19, 63 (1974) [Pis'ma ZhETF 19, 103 (1974)].

21. R.J. Sayer, R.H. Prince, and W.W. Duley, Phys. Status Solidi (b) $\mathbf{1 0 5}, 727$ (1981).

22. O. Oehler, D.A. Smith, and K. Dressler, J. Chem. Phys. 66, 2097 (1977).

23. R.E. Boltnev, E.B. Gordon, V.V. Khmelenko, I.N. Krushinskaya, M.V. Martynenko, A.A. Pelmenev, E.A. Popov, and A.F. Shestakov, Chem. Phys. 189, 367 (1994).

24. R.E. Boltnev, I.B. Bykhalo, I.N. Krushinskaya, A.A. Pelmenev, V.V. Khmelenko, D.M. Lee, I.V. Khyzhniy, S.A. Uyutnov, E.V. Savchenko, A.N. Ponomaryov, G.B. Gumenchuk, and V.E. Bondybey, Fiz. Nizk. Temp. 39, 580 (2013) [Low Temp. Phys. 39, 451 (2013)].

25. I.N. Krushinskaya, R.E. Boltnev, I.B. Bykhalo, A.A. Pelmenev, V.V. Khmelenko, and D.M. Lee, Fiz. Nizk. Temp. 41, 541 (2015) [Low Temp. Phys. 41, 419 (2015)].

26. G.P. Bewley, D.P. Lathrop, and K.R. Sreenivasan, Nature 441, 588 (2006).
27. R.E. Boltnev, I.B. Bykhalo, I.N. Krushinskaya, A.A. Pelmenev, V.V. Khmelenko, S. Mao, A. Meraki, S. Wilde, P.T. McColgan, and D.M. Lee, J. Phys. Chem. A 119, 2438 (2015).

28. W.C. Richardson and D.W. Setser, J. Chem. Phys. 58, 1809 (1973).

29. J.A. Guthrie, R.C. Chaney, and A.J. Cunningham, J. Chem. Phys. 95, 930 (1991).

30. H.H. Hwang, J.K. Olthoff, R.J. Van Brunt, S.B. Radovanov, and M.J. Kushner, J. Appl. Phys. 79, 93 (1996).

31. P. Tosi, W. Lu, D. Bassi, and R. Tarroni, J. Chem. Phys. 114, 2149 (2001).

32. D.K. Bohme, D.B. Dunkin, F.C. Fehsenfeld, and E.E. Ferguson, J. Chem. Phys. 51, 863 (1969).

33. Y.S. Cao and R. Johnsen, J. Chem. Phys. 95, 7356 (1991).

34. P. Stracke, F. Wiegershaus, S. Krischok, and V. Kempter, Surf. Science 396, 212 (1998).

35. N. Lorente, D. Teillet-Billy, and J.-P. Gauyacq, Surf. Science 432, 155 (1999).

36. J. Marbach, F.X. Bronold, and H. Fehske, Eur. Phys. J. D 66, 106 (2012).

37. M. Frankowski, E.V. Savchenko, A.M. Smith-Gicklhorn, O.N. Grigorashchenko, G.B. Gumenchuk, and V.E. Bondybey, J. Chem. Phys. 121, 1474 (2004).

38. E.V. Savchenko, I.V. Khyzhniy, S.A. Uyutnov, G.B. Gumenchuk, A.N. Ponomaryov, and V.E. Bondybey, IOP Conf. Series: Materials Science and Engineering 15, 012082 (2010).

39. O.N. Grigorashchenko, V.V. Rudenkov, I.V. Khizhniy, E.V. Savchenko, M. Frankowski, A.M. Smith-Gicklhorn, M.K. Beyer, and V.E. Bondybey, Fiz. Nizk. Temp. 29, 1147 (2003) [Low Temp. Phys. 29, 876 (2003)].

40. A.A. Radtsig and B.M. Smirnov, Reference Data on Atoms, Molecules, and Ions, Springer, Berlin (1985).

41. G.B. Gumenchuk, M.A. Bludov, and A.G. Belov, Fiz. Nizk. Temp. 31, 237 (2005) [Low Temp. Phys. 31, 179 (2005)].

42. H.P. Broida and R.W. Nicholls, J. Chem. Phys. 32, 623 (1960).

43. R.A. Hemstreet and J.R. Hamilton, J. Chem. Phys. 34, 948 (1961).

44. B. Brocklehurst and G. Pimentel, J. Chem. Phys. 36, 2040 (1962).

45. E.V. Savchenko, I.V. Khyzhniy, S.A. Uyutnov, A.N. Ponomaryov, G.B. Gumenchuk, and V.E. Bondybey, Fiz. Nizk. Temp. 39, 574 (2013) [Low Temp. Phys. 39, 446 (2013)].

46. O. Dutuit, N. Carrasco, R. Thissen, V. Vuitton, C. Alcaraz, P. Pernot, N. Balucani, P. Casavecchia, A. Canosa, S. Le Picard, J.C. Loison, Z. Herman, J. Zabka, D. Ascenzi, P. Tosi, P. Franceschi, S.D. Price, and P. Lavvas, Astrophys. J. Suppl. Ser. 204, 20 (2013).

47. V. Zhaunerchyk, W.D. Geppert, E. Vigren, M. Hamberg, M. Danielsson, M. Larsson, R.D. Thomas, M. Kaminska, and F. Osterdahl, J. Chem. Phys. 127, 014305 (2007).

48. R. Tian, J.C. Facelli, and J. Michl, J. Phys. Chem. 92, 4073 (1988).

49. E.V. Savchenko, I.V. Khyzhniy, S.A. Uyutnov, A.P. Barabashov, G.B. Gumenchuk, M.K. Beyer, A.N. Ponomaryov, and V.E. Bondybey, J. Phys. Chem. A 119, 2475 (2015). 
50. E.V. Savchenko, I.V. Khyzhniy, S.A. Uyutnov, A.P. Barabashov, G.B. Gumenchuk, A.N. Ponomaryov, and V.E. Bondybey, Phys. Status Solidi (c) 12, 49 (2015).

51. E.V. Savchenko and V.E. Bondybey, Phys. Status Solidi (a) 202, 221 (2005).

52. G.B. Gumenchuk, A.N. Ponomaryov, A.G. Belov, E.V. Savchenko, and V.E. Bondybey, Fiz. Nizk. Temp. 33, 694 (2007) [Low Temp. Phys. 33, 523 (2007)].

53. E.V. Savchenko, I.V. Khyzhniy, S.A. Uyutnov, G.B. Gumenchuk, A.N. Ponomaryov, and V.E. Bondybey, Fiz. Nizk. Temp. 35, 520 (2009) [Low Temp. Phys. 35, 409 (2009)].
54. G.B. Gumenchuk, A.N. Ponomaryov, I.V. Khyzhniy, S.A. Uyutnov, E.V. Savchenko, and V.E. Bondybey, Physics Procedia 2, 441 (2009).

55. E.V. Savchenko, I.V. Khyzhniy, S.A. Uyutnov, M.A. Bludov, G.B. Gumenchuk, and V.E. Bondybey, Nucl. Instr. Meth. B (2015).

56. G.B. Gumenchuk, I.V. Khyzhniy, A.N. Ponomaryov, M.A. Bludov, S.A. Uyutnov, A.G. Belov, E.V. Savchenko, and V.E. Bondybey, Fiz. Nizk. Temp. 34, 309 (2008) [Low Temp. Phys. 34, 241 (2008)].

57. J.T. Randall, M.H.F. Wilkins, Proc. R. Soc. Lond. A 184, 390 (1945). 